\section{Practical Procedures in the Management of Tooth Wear}

Authors: Subir Banerji, Shamir B. Mehta,

Niek Opdam, Bas Loomans

Publisher:Wiley-Blackwell, Hoboken, NJ, USA

Language: English

ISBN: 978-1-119-38986-6

Edition: 1/e

Publish Year: 2020

Pages: 248, illustreted

Price: $€ 89.99$

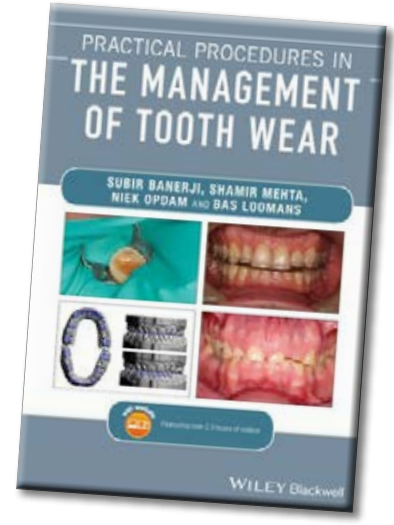

Marian-Vladimi

Constantinescu

DDS, MSc, PhD

Holistic Dental \& Medical Institute

of Bucharest-ROPOSTURO

Bucharest, Romania

e-mail:

dr.vladimir.constantinescu@gmail.com

Currently, in his daily practice, a dentist comes across more and more patients who have tooth wear due to the excessive consumption of carbonated drinks, bruxism and gastric regurgitation. Practical Procedures in the Management of Tooth Wear is a book drafted by a team of international clinical experts which describes a comprehensive number of examples of all practical procedures that address all cases of tooth wear from minimally invasive to invasive methods for treatment. The topics include patient assessment and diagnosis, treatment planning from localized to generalized tooth wear, monitoring, management, and maintenance of tooth wear.

The book has 14 chapters, an index and a companion website access of high-quality instructional videos. The first four chapters address the prevalence, the aetiology and presentation of tooth wear and the clinical assessment and diagnosis of the wear patient, including the use of common clinical indices.

Starting from the occlusion analysis in relation to tooth wear, the book establishes prevention strategies, the role of occlusal splints for patients with tooth wear and treatment planning as well as the application of diagnostic techniques.

The following chapters discuss concepts covering the restoration of worn dentition and present an overview of dental materials selection for the management of tooth wear.

The next three chapters tackle the principles and clinical management of localized anterior and posterior tooth wear, and generalized tooth wear.

The last chapter approaches the prognosis of the restored worn dentition: contingency planning, the importance of maintenance, and recall.

Practical Procedures in the Management of Tooth Wear is a clearly written book, very well illustrated, providing readers with a clinical guide to address dental tooth wear. It is a unique source of information for general dental practitioners, senior undergraduate dental students, and postgraduates which aim to treat this category of patients to high-quality standards.

DOI: http://www.stomaeduj.com 10.25241/stomaeduj.2020.7(1).bookreview.5

The Books Review is drafted in the reviewer's sole wording and illustrates his opinions. 